\title{
Velocity versus Offset (VVO) Estimation Using Local Event Correlation and Its Application in Seismic Processing and Analysis
}

\author{
Supriyono, Awali Priyono, Wahyu Triyoso \& Hilman Mardiyan \\ Graduate Program in Earth Sciences, Bandung Institute of Technology \\ Jalan Ganesha No. 10, Bandung 40132, Indonesia \\ Email: supriyono.itb@gmail.com
}

\begin{abstract}
Conventional velocity analysis is usually done in a relatively spare grid, for instance every half kilometers, during the processing of seismic data. It is very laborious work and very subjective. To deliver an accurate velocity picking, processing geophysicists must have a good understanding of geological background of area being analyzed and experiences. Velocity errors often occur during picking. Proper quality control and checking are a must. A good and reliable velocity field is very important in seismic processing for achieving highquality seismic images as well as for delivering an accurate depth conversion. The new method presented here, was developed to correct velocity errors automatically by means of residual velocity correction, and to produce an offsetdependent RMS velocity field at the same time. The method is data driven, based on the normal move out equation (NMO) and measuring the local even correlation between adjacent traces. The stacking velocity is derived simply by averaging the velocity field. The proposed method was tested on synthetic and real data examples with good result. The velocity field has certain characteristics related to hydrocarbon presence. Author developed a new DHI method using velocity gradient attributes by cross-plotting the velocity versus offset (VVO). The velocity gradient exhibits high anomalous values in the presence of gas.
\end{abstract}

Keywords: automatic velocity analysis; correlation; normal moveout; residual moveout correction; velocity versus offset.

\section{$1 \quad$ Introduction}

Velocity analysis is one of the critical steps in seismic data processing. Such an analysis is applied to a velocity semblance panel manually and interactively on the CMP gather according to an approximation of the NMO equation, as originally proposed by Taner and Koehler [1]. The stacking velocity is determined in a relatively sparse grid and randomly based on the highest velocity semblance picked from top to bottom. The velocity field is then interpolated linearly from time zero to record length.

Received February $7^{\text {th }}, 2012$, Revised February $7^{\text {th }}, 2013$, Accepted for publication February $7^{\text {th }}, 2013$. 
The above analysis is repeated spatially across the seismic line, or within a regular grid for 3D data. The distance between velocity analysis locations is usually subject to the client's request. Careful quality control of velocity picking on the semblance and NMO corrected gathers are normal procedure in the processing center in order to derive an accurate and reliable velocity field for further processing steps.

An accurate velocity is very important, specifically for the stacking process to generate migrated stacks or volumes as final deliverables for clients that will be used for interpretation. The stacking or RMS velocity field can be used to develop a velocity model after being calibrated to well data for time to depth conversion.

An automatic residual moveout correction tool is now available in the market and its use has become standard procedure in the processing center to help processing geophysicists prepare the final velocity field for stacking. It is based on travel-time approximations and semblance coherency of the entire CMP gather. The far offset is used as a reference in order to calculate the correct RMS velocity since it provides the highest residual.

A set of stacking velocities is scanned along approximated travel time equations across the offsets. The highest semblance value is automatically selected as the corrected Vrms. McCowan and Graul [2] measure the residual moveout at far offset as reference to calculate the correct Vrms to flatten the CMP gather. This method only outputs a single velocity function for each CMP gather. It will fail when the input CMP gathers exhibit an anisotropic phenomenon, where velocity at the far offset (higher angle) is higher than that at normal incident.

A new method for calculating the correct Vrms automatically was developed without scanning the set of velocities, but by considering only the local even correlation of adjacent traces from the nearest to far end offset. The proposed method honors changes of velocity in each offset. Therefore, any anisotropic characters in the CMP gather will be measured automatically. This principle is simple, but requires high signal-to-noise-ratio data and good event continuity along the offsets within the CMP gather.

This requirement is achievable by applying signal coherency enhancement and noise reduction in the preparation stage to the input gather. Other noise filtering is applied to intermediate products (residual move-out for multiples) to remove outliers that fall beyond the trend. This method is tested using model and real data examples. It works well. 


\section{Theory}

The seismic stacked section, either the product of the stacking process, prestack time migration or pre-stack depth migration, does not necessarily have a good image. It is heavily affected by the velocity function being applied to the data. If the velocity is accurate, the reflections in the CMP gather are perfectly flat. Inaccuracy of the migration velocity field leads to the reflections in the image gather not being flat (under- or over-corrected). The residual moveout or non-flatness of reflections in the CMP gather can be used to calculate the correct RMS velocity field by means of a residual velocity analysis.

Consider a CMP gather after pre-stack time migration. The original subsurface reflection time from a certain offset $(T x)$ is moved to its midpoint position at two-way time $T o$. In order to calculate the moveout correction of the stacking velocity (Vnmo), travel time can be formulated using a Dix equation.

$$
\begin{aligned}
& T_{X}^{2}=T_{0}^{2}+\left[\frac{X}{V_{n m o}}\right]^{2} \\
& \Delta T \approx \frac{1}{2 T_{0}}\left[\frac{X}{V_{n m o}}\right]^{2}
\end{aligned}
$$

where $\Delta T=T x-T o$ and approximating $T x+T o \sim 2 T o . \Delta T$ is the difference between the two-way travel times at offset $X$ and at midpoint or zero offset. This is the so-called moveout correction. The Vnmo is the true RMS velocity (Vnmo is usually equal to Vrms) that corrects seismic reflection events in the CMP gather to become flat after the normal moveout correction process.

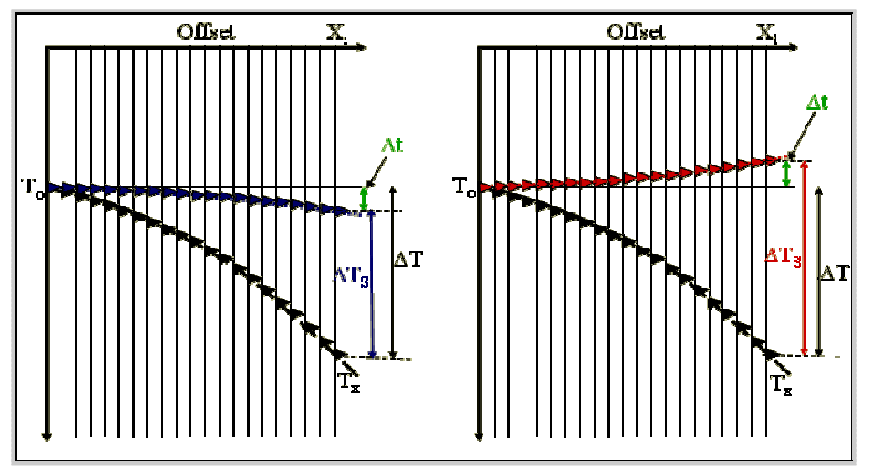

Figure 1 A CMP gather with two-way travel time of reflected signal $T x$ after normal moveout correction with initial incorrect stacking velocity Vstk. On the left for an under-corrected case and on the right for an over-corrected case. 
In the case of the stacking velocity or migration velocity for PSTM being incorrect $(V s t k \neq V n m o$ ), the reflection events are not flat; they curve downward or upward as illustrated in Figure 1. There is a residual moveout or RMO $(\Delta t)$ that needs to be calculated and corrected. The RMO is the time difference between the two-way time after correction using the initial stacking velocity (Vstk) and that of using the correct Vnmo.

The sign of the residual moveout is positive if the reflection curve is facing downward due to the stacking velocity being relatively higher than the correct NMO velocity $(V s t k>V n m o)$. One has to remember that travel time increases downward in a seismic display. On the other hand, the residual moveout will be negative if the reflection curve is facing upward or the stacking velocity applied to the gather is relatively low compared to the correct NMO velocity $(V s t k<V n m o)$.

Mathematically, the residual moveout in Figure 1 can be expressed by the following formula:

$$
\Delta t=\Delta T-\Delta T_{S}
$$

where, $\Delta T s$ is the normal moveout correction with initial incorrect stacking velocity Vstk which is not equal to Vrms.

\subsection{Approximation Solution of Offset-Dependent RMS Velocity}

From Eq. (2) above, moveout correction is calculated using the following approximation formula:

$$
\Delta T_{S} \approx \frac{1}{2 T_{0}}\left[\frac{X}{V_{S t k}}\right]^{2}
$$

By combining Eqs. (1), (2) and (3), it is very straightforward to derive the relationship between the residual moveout $(\Delta t)$ resulted from the application of the initial stacking velocity (Vstk) to the CMP gather for normal moveout correction, compared to normal moveout correction with the true RMS velocity (Vrms), which flattens the reflection events in the gather.

$$
\Delta t \approx \frac{1}{2 T_{0}}\left[\frac{X}{V_{r m s}}\right]^{2}-\frac{1}{2 T_{0}}\left[\frac{X}{V_{S t k}}\right]^{2}
$$

Eq. (5) specifically indicates that the residual moveout is a function of offset $X$ as well as the stacking and RMS velocities. Every offset position $\left(X_{j}\right)$ has its own residual moveout value $\left(\Delta t_{j}\right)$. Therefore, the normal moveout velocity for 
each individual offset position within the CMP gather can be approximated using the following equation:

$$
\Delta t_{j} \approx \frac{X_{j}^{2}}{2 T_{0}}\left[\frac{1}{V r m s_{j}^{2}}-\frac{1}{V_{S t k}^{2}}\right]
$$

where $\mathrm{Vrms}_{j}$ is the correct normal moveout velocity at position $X_{j}$ and moveout time $T o$. The equation above can be rearranged in the form of slowness.

$$
\frac{1}{V r m s_{j}^{2}} \approx \frac{1}{V s t k_{j}^{2}}+\frac{2 T_{0} \Delta_{j}}{X_{j}^{2}}
$$

All variables in Eq. (7) are known. $T_{o}$ is the travel time being analyzed, while $V s t k$ is the initial stacking velocity applied to the input CMP gather. The residual moveout $\Delta t_{j}$ is analyzed through cross-correlation of each trace with the nearest offset. Therefore the correct NMO velocity of each offset can be calculated.

\subsection{Exact Solution of Offset Dependent RMS Velocity}

The derivation of the exact solution is started from the same travel-time Eq. (1). At a certain offset position $X_{j}$, the corresponding two-way travel time is written in form of:

$$
\begin{aligned}
& T x_{j}^{2}=T_{0}^{2}+\left[\frac{X_{j}}{V r m s_{j}}\right]^{2} \text { or } T x_{j}=\left(T_{0}^{2}+\left[\frac{X_{j}}{V r m s_{j}}\right]^{2}\right)^{1 / 2} \\
& T x_{j}-T_{0}=\Delta T=\left[T_{0}^{2}+\left(\frac{X_{j}}{V r m s_{j}}\right)^{2}\right]^{1 / 2}-T_{0}
\end{aligned}
$$

Vrms is the correct velocity and is initially constant for all offsets. The normal moveout correction with this velocity will move the reflection from all offsets to the same position as normal incident. There are two possible cases.

\subsubsection{Under-corrected Case}

Under-correction happens if the initial stacking velocity Vstk used in the normal moveout correction is higher than the true RMS velocity (Vstk>Vrms). Vstk is known as the input velocity in the NMO process, but Vrms is unknown, i.e. to be calculated. Mathematically, the moveout shift of the NMO correction using velocity Vstk is written as follows: 


$$
\Delta T_{s}=\left[T_{0}^{2}+\left(\frac{X}{V s t k}\right)^{2}\right]^{1 / 2}-T_{0}
$$

Similarly, the correct moveout occurs if Vrms is used in the NMO correction.

$$
\Delta T=\left[T_{0}^{2}+\left(\frac{X}{V r m s}\right)^{2}\right]^{1 / 2}-T_{0}
$$

Referring to Eq. (3), the residual moveout at a certain offset position $X_{j}$ equal to $\Delta t_{j}$ is a time-shift difference between the NMO correction using Vrms and using Vstk. Vrms is still unknown. Meanwhile $\Delta t_{j}$ is observed from the NMO'ed CMP gather and can be calculated by subtracting Eq. (9) from Eq. (10).

$$
\Delta t_{j}=\left[T_{0}^{2}+\left(\frac{X_{j}}{V r m s_{j}}\right)^{2}\right]^{1 / 2}-\left[T_{0}^{2}+\left(\frac{X_{j}}{V_{s t k}}\right)^{2}\right]^{1 / 2}
$$

Please note that all variables in the second component of Eq. (11) are known. To is the two-way time of the trace being analyzed. If we consider the second component equal to $d_{j}$, then the above equation becomes:

$$
\begin{aligned}
& \Delta t_{j}+d_{j}=\left[T_{0}^{2}+\left(\frac{X_{j}}{V r m s_{j}}\right)^{2}\right]^{1 / 2} \\
& \frac{1}{V r m s_{j}^{2}}=\frac{\left(\Delta t_{j}+d_{j}\right)^{2}-T_{0}^{2}}{X_{j}^{2}}
\end{aligned}
$$

Eq. (13) above is the exact solution that relates $V r m s_{j}$ and offset $X_{j}$, and is computed as a function of the residual moveout at offset $\Delta t_{j}$. Each offset position will have its corresponding RMS velocity. This phenomenon is called offset dependent RMS velocity.

\subsubsection{Over-corrected Case}

Over-correction happens when the initial stacking velocity Vstk is lower than the true RMS velocity $(V s t k<V r m s)$. We use the same definition of residual moveout as mentioned in the under-corrected case, $\Delta t=\Delta T-\Delta T s$. The mathematical formulation is similar, but the value is negative, since $\Delta T s$ is larger than $\Delta T$. The offset-dependent residual moveout follows Eq. (11), with 
the second component being larger. Using a similar derivation, the offsetdependent RMS velocity for the over-corrected case leads to Eq. (13).

This result leads to the conclusion that the equation for calculating the offsetdependent RMS velocity from seismic data is a generic formula. It is applicable for all conditions of input data, under- or over-corrected. The input data must be CMP gathers after normal moveout correction with initial stacking velocities. It does not require an accurate initial stacking velocity to start with. The computer can calculate the correct Vrms automatically.

\section{Methodology and Validation}

The objective of the methodology developed in this paper is to estimate the offset-dependent RMS velocity $\operatorname{Vrms}(t, x)$ using local event correlation (LEC). Duveneck and Traub [3] used this method to perform automatic moveout correction to flatten each reflection in the CMP gather to its calculated To from event correlations, where $T o$ is the projected normal incident two-way time. Input data is anun-NMO'ed CMP gather, while the output is expected to be a 'flat' CMP gather. All samples in the input data are mapped to To using a kind of interpolation. No RMS velocity field is produced.

In the method I propose here, the input data is a CMP gather after application of normal moveout using initial stacking velocities. The initial stacking velocities do not need to be accurate. However, a more accurate stacking velocity is better for estimating the RMS velocity in far offsets. The computation is based on the assumption that the nearest offset is perfectly corrected during the normal moveout process. The nearest offset is selected to be the reference trace to estimate the RMS velocities at all offsets within the CMP gather. The output are offset-dependent RMS velocities for all samples or velocity gathers $\operatorname{Vrms}(t, x)$.

The concept is simple. For each data sample on a given trace in the CMP gather with its two-way time at $T o$, a local time window is defined. The reference time being analyzed $(T o)$ is in the middle of the window. The window length must be larger than one wavelength in order to capture the reflection signal in that trace. 80 milliseconds of window length is sufficient, and it is used for this purpose. The seismic trace within a given offset and time window is then cross-correlated with the previous offset within the same time window.

Cross-correlation starts from the second offset $\left(X_{1}\right)$, with the nearest offset $(X o)$ as reference. The time delay is derived from the maximum cross-correlation coefficient. It is a measure of the residual moveout $(\Delta t)$ of the second offset relative to the nearest offset. The time delay computed from the crosscorrelation between the second offset and the first offset is equal to the absolute 
residual moveout of the second offset. A fast Fourier transform can be used to speed up computation, as well as allowing a smaller time delay than the sampling interval.

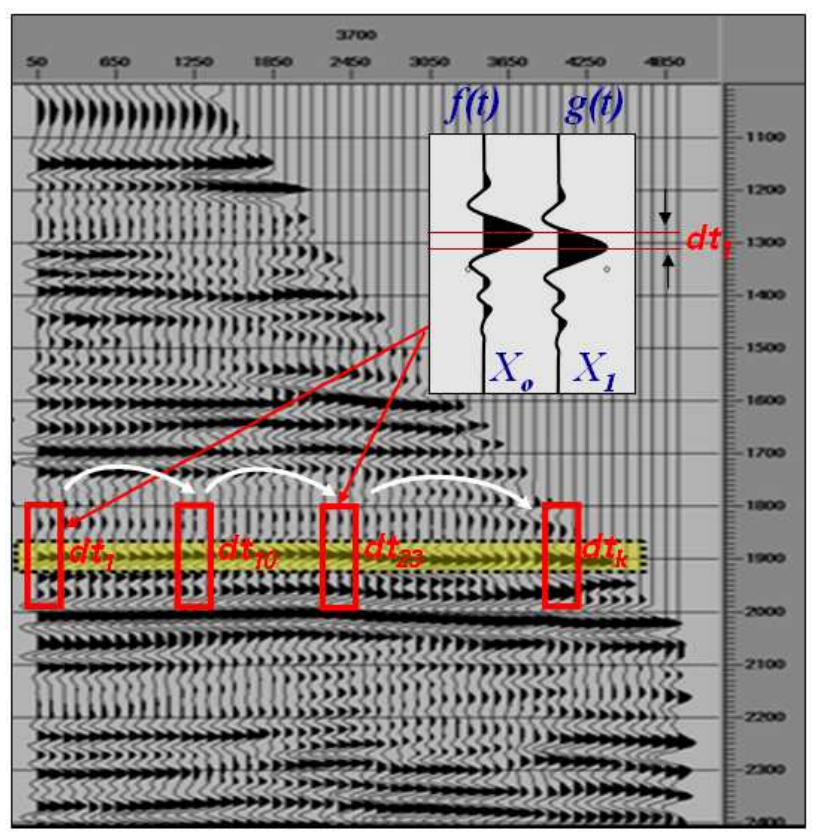

Figure 2 Local event correlation of two adjacent traces.

The maximum cross-correlation coefficient is automatically selected, representing the time delay of the second offset $\left(d t_{1}\right)$ relative to the first one. Cross-correlation is then moved between offset three against offset two. This process is repeated until the final offset and the corresponding time delay $\left(d t_{f}\right)$ have been derived, see Figure 2.

The computation is moved to the next window analysis. The time step of analysis is controlled by the vertical resolution target that needs to be achieved, i.e. $20 \mathrm{~ms}, 40 \mathrm{~ms}$, etc. After completion of this process in both directions, i.e. horizontal (offset) and vertical (two-way time), a time-delay matrix is produced. If necessary, a moving window median filter is applied to remove outliers due to noise or event discontinuity within the correlation window in the input CMP gather.

Please note that $d t_{k}$ and $\Delta t_{k}$ are different. The first is the time delay between the $k^{\text {th }}$ offset relative to the previous offset $(k-1)$. While the latter is the absolute time delay of the $k^{\text {th }}$ offset (relative to the $1^{\text {st }}$ offset), and is assumed to be the 
residual moveout of the $k^{\text {th }}$ offset for that particular time window. The relationship between both quantities is defined as follows:

$$
\Delta t_{k}=\sum_{i=1}^{k} d t_{i}
$$

Another critical factor in this method is the design correlation window movement followingthe curvature of the reflection events from near to far offsets. If the initial stacking velocity is good, the window's movement in the horizontal direction toward far offset follows a flat reflection. However, when the initial velocities are not accurate, the movement must follow the reflection curvature. The window length is kept constant. The start time changes across the offset. The change in window start time is controlled by the delay time from the previous local cross-correlation.

$$
T w_{k+1}=T w_{k}+d t_{k}
$$

where $T w$ is the window start time. Figure 3 illustrates the correlation window between adjacent traces from the nearest until far offset (top). The time window for local event correlation shifts upward from near to far offset. The correlation windows capture the same seismic characters from the same subsurface reflections. The corresponding residual moveout is calculated for each offset using Eq. (14), plotted in a solid line.

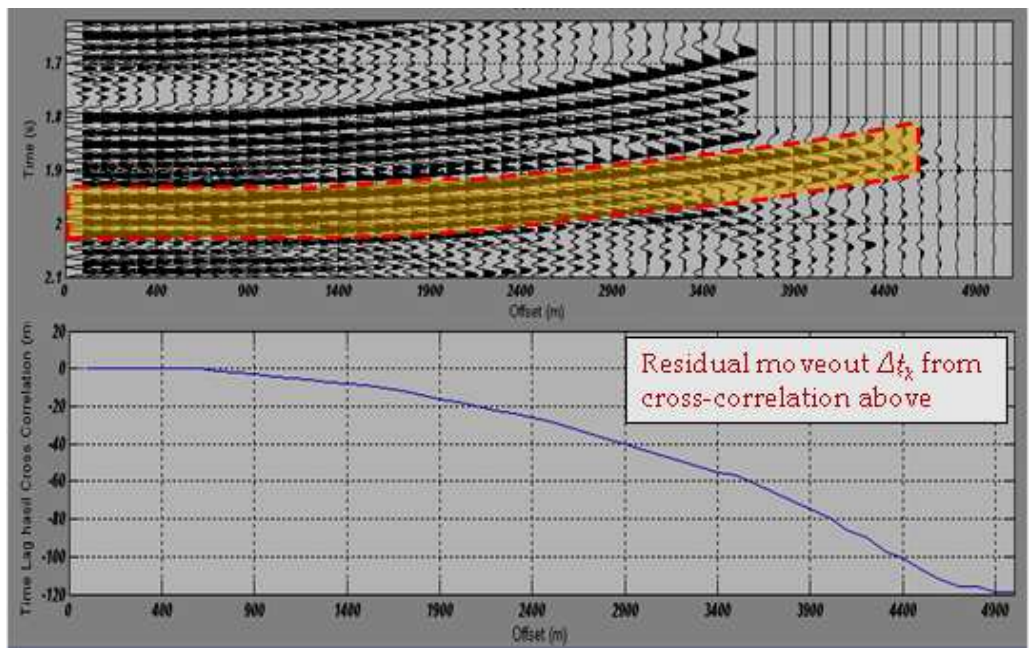

Figure 3 Correlation window moves across the offset following reflection events in shaded polygon.

Input data must be NMO-corrected with initial stacking velocities. They do not need to be accurate, but the more accurate they are, the better. Application of 
this method to a CMP gather is a kind of data transformation from amplitude gather $A(t, x)$ to velocity gather $\operatorname{Vrms}(t, x)$. The output velocity gather is then stacked (average across the offsets) to produce a high-density RMS velocity field that will be used for normal moveout correction and the stacking process.

To validate the accuracy of this method, we have applied it to a synthetic CMP gather with normal moveout correction using $90 \%$ (blue line in Figure 4) and $110 \%$ (green line in Figure 4) of the RMS velocities, representing the errors in the initial stacking velocities. The velocity scanning code was run to both CMP gathers as input. Two calculated RMS velocities were derived: Vrms_calc $c_{l}$ is the calculated Vrms from the over-corrected input gather, and Vrms_calc $c_{2}$ is the calculated Vrms from under-corrected input gather.

Figure 4 shows a comparison of the calculated Vrms from the over- and undercorrected input gathers. The first panel (left) is the CMP gather after NMO correction using the calculated RMS velocity Vrms_calc $c_{1}$ from the overcorrected input gather (90\% Vrms), denoted with red line. The second panel (middle) is the CMP gather after NMO correction with the calculated RMS velocities $\mathrm{Vrms}_{-} \mathrm{Calc}_{2}$ from the under-corrected input gather (110\% Vrms), denoted with brown line. Both gathers are quite flat, which means that the calculated Vrms in both cases converges towards the correct Vrms, regardless of the initial conditions of the input CMP gathers.
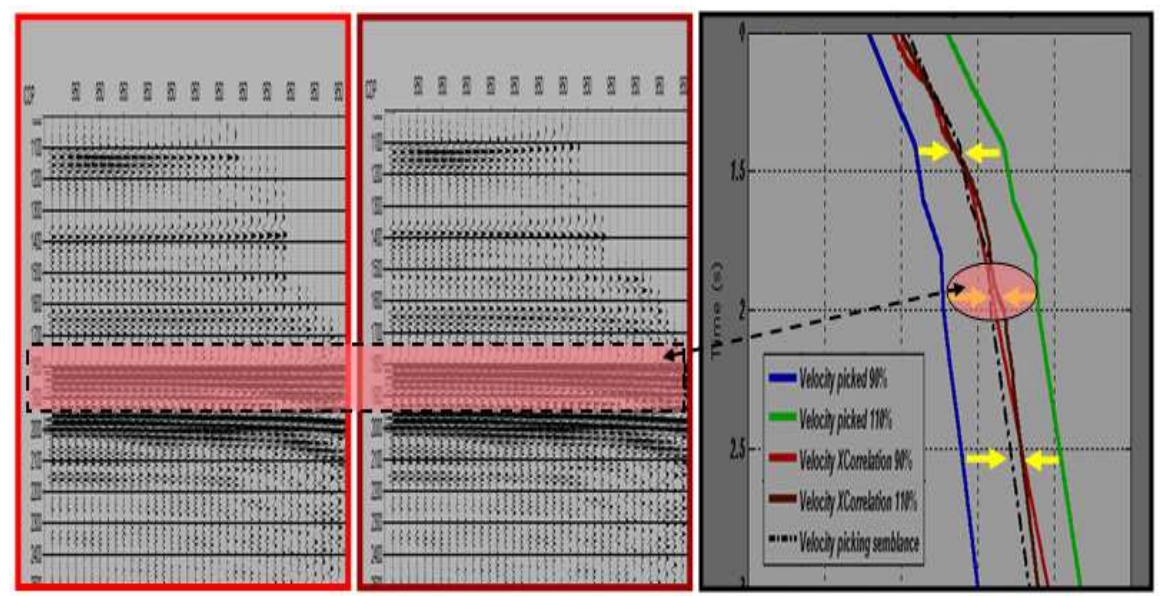

Figure 4 Code validation test result on a synthetic CMP gather using initial NMO correction $90 \%$ and $110 \%$ of RMS velocities.

The third panel (right) is a plot of the RMS velocities. The dotted black line in the middle is the correct Vrms derived from conventional velocity picking. The 
solid blue and green lines are the $90 \%$ and $110 \%$ RMS velocities. The calculated Vrms_calc $c_{1}$ is plotted in a red line, while Vrms_calc $c_{2}$ is plotted in a brown line. It is clear from the graphs that the correct Vrms and its estimation using this method are close together. The difference is very small, because the NMO'ed gathers using these velocities are quite similar. The calculated RMS velocities mentioned above (Vrms_calc $c_{1}$ and Vrms_calc $c_{2}$ ) are actually the average of the calculated RMS velocities from each individual offset within the CMP gather, $\operatorname{Vrms}(t, x)$.

Figure 5 shows velocity scanning across the offset using the LEC method. The lowest panels are plots of the $\operatorname{Vrms}(t, x)$ field from the same sandstone reservoir. The middle panels contain graphs of the associated residual moveout computed within the window, drawn in yellow polygons.

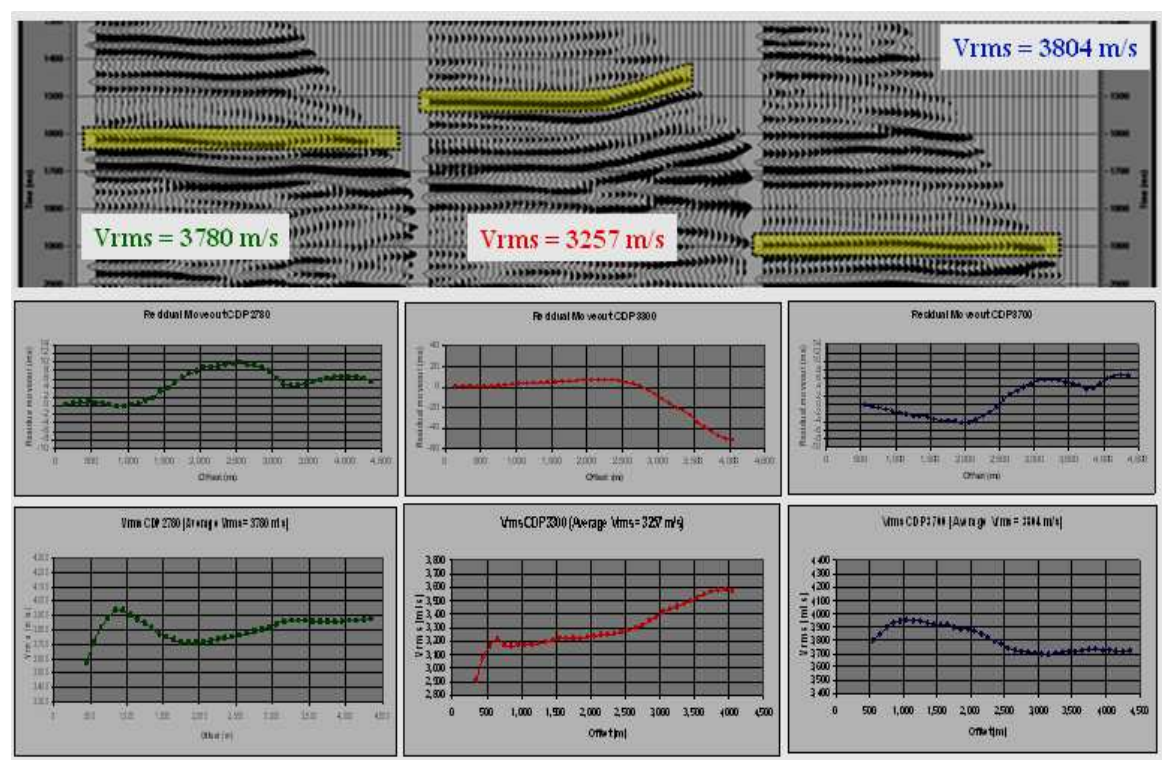

Figure 5 Scanning of RMS velocity along the offset $\operatorname{Vrms}(t, x)$ using the LEC method proposed in this paper.

Later, the velocity gathers $\operatorname{Vrms}(t, x)$ will be used for another purpose. In a previous study, Supriyono [4] proposed a new velocity attribute extracted from the velocity gather that is relatively sensitive to the presence of gas. In the reflectivity data, one can produce the intercept and amplitude gradient during AVO analysis for hydrocarbon prediction. Using the same analogy, the intercept and velocity gradient are derived from the velocity gathers. The intercept is the vertical velocity. Meanwhile, the velocity gradient indicates an anisotropic phenomenon. One of the anisotropic sources in sedimentary rock is fluid 
content (gas). The presence of gas in weak anisotropic sandstone significantly increases its anisotropic properties, according to Bandyopadhyay [5]. For this reason, the velocity gradient is calculated as an alternative attribute for direct hydrocarbon indication, in addition to AVO, as suggested by Supriyono [6].

\section{$4 \quad$ Application to Real Data}

This method was tested on real data and compared to other automatic velocity scanning method, such as the $T^{2}-X^{2}$ method. The seismic data are located offshore from an open area in the Asia Pacific and have a relatively complex structure, so we can evaluate the stability of the proposed method. Data processing applied to the data is simple, consisting of: amplitude recovery, noise attenuation, and deconvolution. The test was done on un-migrated gathers, after multiple suppresion by radon transform.

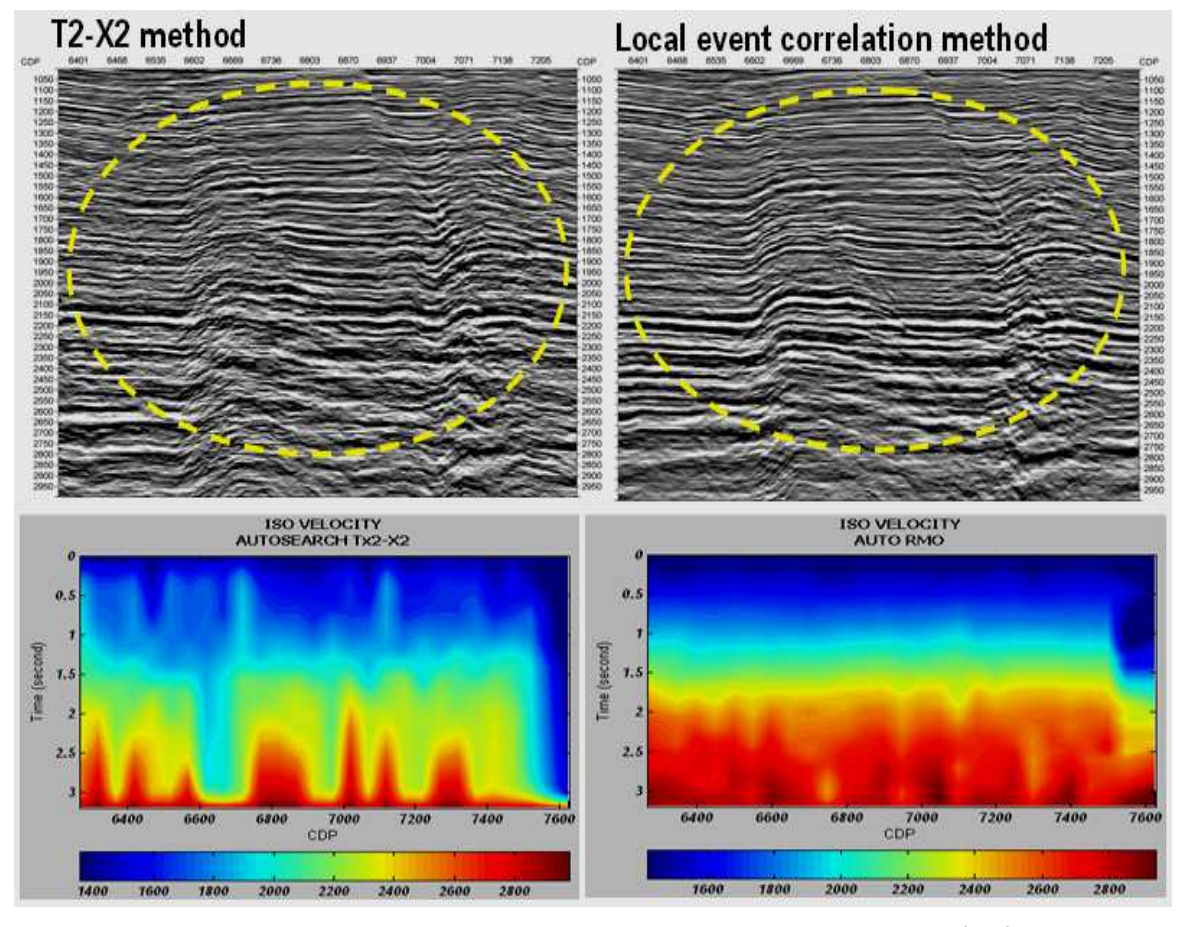

Figure 6 Stack section using Vrms derived from the automatic $T^{2}-X^{2}$ method (upper left). Stack section using Vrms from the local event correlation method (upper right). Corresponding velocity fields are displayed below its stacked sections. 
The velocity fields and stacked sections from both methods are displayed side by side for comparison in Figure 6 . On the left side are the stacked section and the RMS velocity field from the $T^{2}-X^{2}$ method, while the local event correlation method is in the right panel. The stacked section resulted from local event correlation shows superior results in whole parts of the seismic section, more focus and a sharper image, also in the faulting and folding areas as seen on the seismic characters inside the dotted yellow ellips. Diffractions are also better preserved in the LEC method.

The $T^{2}-X^{2}$ method fails to delineate a detailed velocity field in the area of complex structures, for example in the proximity of faulting and folding. Below 1.5 seconds twt, the velocity radically changes up and down. This doesn't reflect the geological structures seen in the seismic section.

Another test was applied to a marine dataset from a different area in the same region, with the objective to compare the Vrms field computed by the LEC method with the Vrms derived from manual picking during the velocity analysis. The input dataset was a pre-migration intermediate CMP gather from a processing contractor, with multiple suppression by radon transform, and additional noise cleanup. Preconditioning is very critical in the LEC method to prepare clean input datasets in order to improve stability and accuracy in determining delay time.

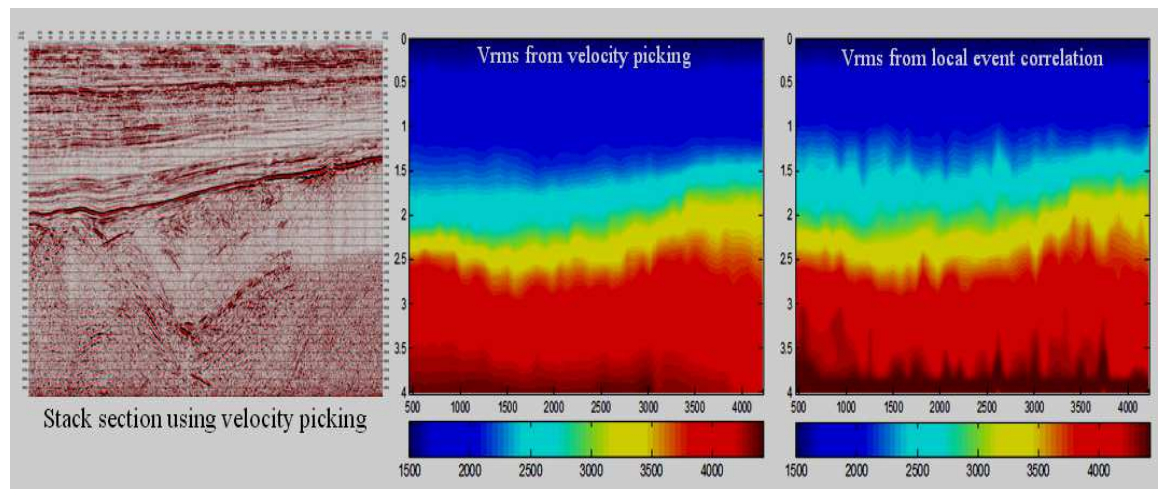

Figure 7 Comparison of Vrms fields from conventional velocity analysis picking (middle panel) and Vrms field derived from local event correlation method (right panel). Corresponding stacked section is in the left panel.

The stacked sections using both velocity fields are visually identical. The left panel in Figure 7 is the stack display of this line. The Vrms field automatically computed by the LEC method (right panel) shows similar values and trends compared to the velocity from manual picking (middle panel). It is logical that 
the seismic sections look identical. The Vrms field derived from the LEC method captures small changes in velocity variation in spatial directions. This variability can actually be seen in the Vrms from manual picking as well, but with a smaller magnitude due to the smoothing effect from sparse picking in thevertical direction.

\section{Conclusion}

We have demonstrated the application on real data examples of the LEC method to derive Vrms as the result of an automatic estimation of offset-dependent RMS velocity $\operatorname{Vrms}(t, x)$. The Vrms field is the average of $\operatorname{Vrms}(t, x)$ across the offset. It is assumed that the near-offset trace is close to zero offset, and can be used as the reference trace. The input data must be NMO-corrected and clean of multiples and noise to increase computation stability. Prior to calculation of the Vrms on each offset, intermediate time delays scanned from the LEC are filtered to remove outliers.

The Vrms field produced by the LEC method is a high-resolution velocity field. The resolution is controlled by increments of local window correlation in the vertical direction. The computation can be made to all CMP gathers. This highresolution velocity field is very useful, for instance for developing a velocity model for depth conversion and for pore pressure prediction.

LEC is a robust method for estimating Vrms from CMP gathers. The resulted velocity field is consistent, and is close to the actual Vrms, regardless if the input gather is over-corrected or under-corrected.The NMO correction and stacking process using actual Vrms from manual velocity picking and Vrms computed by the LEC method produce almost identical results. The initial stacking velocity applied to the input gather does not need to be perfect. Local event correlation will automatically correct the errors.

A requirement of the LEC method is that the input CMP gathers must be clean of noise and multiples, to avoid any misleading in the computation of the delay times and controlling the movement of the local time windows along the offset. This method is based on NMO travel time equation. A pre-stack time migration is suggested for more complex geologies to improve input data quality and computation stability.

\section{Nomenclature}

$\begin{array}{ll}A(t, x) & =\text { Amplitude data in CMP gather } \\ C M P & =\text { Common Mid Point, a surface binning of pre-stack data } \\ d t & =\text { Delay time between adjacent traces }\end{array}$




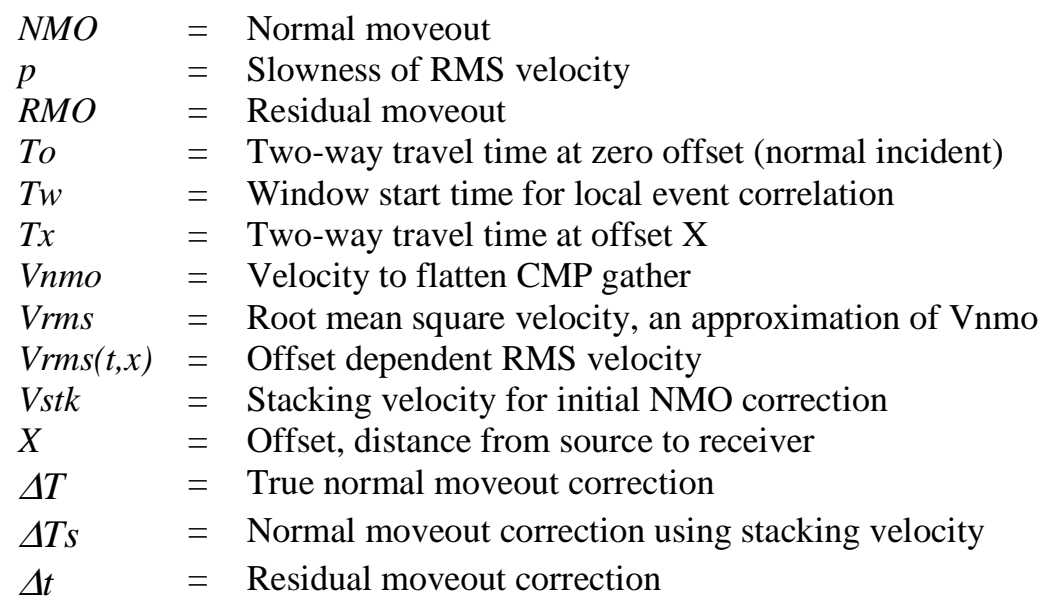

\section{References}

[1] Taner, M.T. \& Koehler, F., Velocity Spectra-Digital Computer Derivation and Applications of Velocity Functions, Geophysics, 34, pp. 859-881, 1969.

[2] McCowan, D.W. \& Graul, J.M., Correcting an RMS Velocity Function Directly from Measurements of Residual Normal Moveout, SEG Technical Program Expanded Abstracts, pp. 3100-3104, 2006.

[3] Duveneck, E. \& Traub, B., Automatic Moveout Correction by Local Event Correlations on Coherency-Enhanced Gathers, SEG Technical Program Expanded Abstracts, pp. 3036-3040, 2006.

[4] Supriyono, Velocity Variation with Angle or Offset (VVO), A New Tool for Direct Hydrocarbon Indicator: Case Studies in Low and High Impedance Clastic Reservoirs, $\mathrm{PhD}$ dissertation, Department of Geophysical Engineering, Bandung Institute of Technology, Bandung, 2011.

[5] Bandyopadhyay, K., Seismic Anisotropy: Geological Causes and Its Implication to Reservoir Geophysics, $\mathrm{PhD}$ dissertation, Department of Geophysics, Standford University, Standford, 2009.

[6] Supriyono, Priyono, A., Triyoso, W. \& Presetya, L., Velocity versus Offset (VVO): A New Tool for Direct Detection of Gas in Low Impedance Sand Reservoirs, First Break EAGE's Journal (European Association of Geoscientists \& Engineers), 30, pp. 59-68, 2012. 